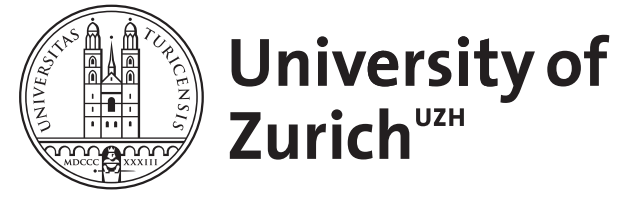
Archive

University of Zurich

University Library

Strickhofstrasse 39

CH-8057 Zurich

www.zora.uzh.ch

Year: 2016

\title{
CME-Labor 47: Monoklonale Gammopathien
}

Annema, Wijtske ; von Eckardstein, Arnold

DOI: https://doi.org/10.1024/1661-8157/a002479

Posted at the Zurich Open Repository and Archive, University of Zurich

ZORA URL: https://doi.org/10.5167/uzh-129369

Journal Article

Accepted Version

Originally published at:

Annema, Wijtske; von Eckardstein, Arnold (2016). CME-Labor 47: Monoklonale Gammopathien. Praxis, 105(19):1123-1124.

DOI: https://doi.org/10.1024/1661-8157/a002479 


\section{CME-Labor 47}

\section{Monoklonale Gammopathien}

\section{Frage 1}

Welche Aussagen zur monoklonalen Gammopathie unbestimmter Signifikanz (MGUS) treffen zu? (Mehrfachauswahl, mehrere Antworten richtig)

a) Die MGUS kommt bei älteren Menschen relativ selten vor.

b) Frauen sind häufiger von einer MGUS betroffen als Männer.

c) Die MGUS hat eine höhere Inzidenz bei Menschen mit schwarzer Hautfarbe als bei Kaukasiern.

d) Diagnostische Kriterien für MGUS sind weniger als $10 \%$ klonale Plasmazellen im Knochenmark und weniger als 30 g/l M-Protein im Serum.

e) Die Mehrzahl der Patienten mit MGUS entwickelt innerhalb von 10 Jahren nach der Diagnose eine maligne Plasmazell-proliferative Erkrankung.

\section{Antworten c) und d) sind richtig}

\section{Kommentar}

Die monoklonale Gammopathie unbestimmter Signifikanz (MGUS) ist charakterisiert durch eine übermässige Produktion von monoklonalem Immunglobulin (M-Protein) ohne weitere klinische Symptomatik. Nicht selten ist die Diagnose ein Zufallsbefund im Rahmen einer Routineuntersuchung. Im hohen Lebensalter kommt die MGUS relativ häufig vor. Gemäss einer amerikanischen Studie beträgt die Inzidenz für die Gruppe von über 50-Jährigen ca. 3\%, in der Gruppe der über 70-Jährigen steigt die Inzidenz auf ca. 5\% [1]. Männer sind etwas häufiger betroffen als Frauen (1.4:1) und in den USA ist die Inzidenz im afroamerikanischen Teil der Bevölkerung doppelt so hoch wie unter Kaukasiern [2]. Die aktuelle Klassifikation der International Myeloma Working Group definiert die MGUS als den Nachweis von weniger als 30 g/l M-Protein im Serum und weniger als 10\% Plasmazellen im Knochenmark (Tab. 1) [3]. Ausserdem dürfen zur Diagnose einer MGUS keine Gammopathie-assoziierten Endorganschäden (z.B. Hyperkalziämie, Niereninsuffizienz, Anämie und Knochenläsionen) oder Amyloidose vorliegen. Die Wahrscheinlichkeit einer Progression einer MGUS zu einem Multiplen Myelom liegt durchschnittlich nur bei 1.5\% pro Jahr [1]. Bei Personen mit monoklonalem IgA oder IgM ist das Risiko eine maligne Erkrankung zu entwickeln höher als bei Personen mit monoklonalem IgG [4]. Daneben hängt das Progressionsrisiko von der Konzentration an M-Protein ab: liegt die M-Protein-Konzentration über $15 \mathrm{~g} / \mathrm{l}$ steigt das 
Risiko um das 2-fache an [4]. Schliesslich geht ein verändertes Verhältnis der freien Leichtketten Kappa und Lambda zueinander mit einer schlechten Prognose einher [5].

Tab 1. Diagnosekriterien der International Myeloma Working Group für die monoklonale Gammopathie unbestimmter Signifikanz (MGUS) [3].

M-Protein im Serum <30 g/l

Klonale Plasmazellen im Knochenmark <10\% der kernhaltigen Zellen

Keine Endorganschäden, wie Hyperkalziämie, Niereninsuffizienz, Anämie und Knochenläsionen (CRAB) oder Amyloidose

\section{Frage 2}

Ein 68-jähriger Mann konsultiert den Hausarzt wegen nicht erklärbarer starker Rückenschmerzen. Er gibt an in den letzten Monaten mehrmals krank gewesen zu sein. Die Serumprotein-Elektrophorese zeigt das Vorhandensein eines M-Gradienten in der GammaFraktion. Mittels Immunfixation kann eine monoklonale Gammopathie IgG-Kappa nachgewiesen werden. Welche weiteren Laboruntersuchungen sind bei diesem Patienten indiziert? (Mehrfachauswahl, mehrere Antworten richtig)
a) Hämoglobin
b) Kreatinin
c) Calcium
d) Laktatdehydrogenase
e) $\beta 2$-Mikroglobulin

\section{Alle Antworten sind richtig}

\section{Kommentar}

Typische klinische Manifestationen des multiplen Myeloms sind Müdigkeit, Knochenbeschwerden, typischerweise an Wirbelsäule oder Brustkorb, und eine erhöhte Infektanfälligkeit. Zur Diagnosestellung eines multiplen Myeloms müssen folgende Kriterien erfüllt sein: der Nachweis von mehr als 10\% klonale Plasmazellen im Knochenmarkaspirat und/oder Biopsie-geprüftes Plasmazytom sowie das Vorliegen einer myelom-bedingten Endorganschädigung [3]. Die Feststellung einer Endorganschädigung erfolgt beim multiplen Myelom gemäss der von der International Myeloma Working Group festgelegten sogenannten CRAB-Kriterien (Tab.2) [3]. Diese enthalten Hyperkalziämie, Niereninsuffizienz, Anämie und osteolytische Knochenläsionen. Nach den aktualisierten Diagnosekriterien der International Myeloma Working Group liegt ebenfalls ein multiples Myelom vor, wenn 
mindestens eine der folgenden myelom-definierende Ereignisse besteht: $\geq 60 \%$ klonale Plasmazellen im Knochenmark, ein Verhältnis der beteiligten/unbeteiligten freien Leichtketten im Serum $\geq 100$ oder mehr als eine fokale Knochenläsion im MRT [3]. Häufig findet man bei einem multiplen Myelom neben erhöhten Kalzium- und Kreatininwerten und erniedrigten Hämoglobinwerten auch eine Erhöhung des Gesamtproteins im Blut, eine Beschleunigung der Blutsenkungsreaktion (bedingt durch die Hypergammaglobulinämie) und seltener eine Thrombozytopenie. In einzelnen Fällen führt die Überproduktion von monoklonalen Immunglobulinen zu einem Hyperviskösitätssyndrom mit Symptomen wie Kopfschmerzen, Sehstörungen, Schwindel und Schleimhautblutungen.

Tab 2. CRAB-Kriterien [3].

\begin{tabular}{|l|l|l|}
\hline \multicolumn{2}{|l|}{ CRAB-Kriterium } & Definition \\
\hline C (Calcium) & Hyperkalziämie & $\begin{array}{l}\text { Kalziumkonzentration im Serum }>2.75 \mathrm{mmol} / \mathrm{l} \text { oder }>0.25 \\
\mathrm{mmol} / \mathrm{l} \text { über der oberen Grenze des Normwertes }\end{array}$ \\
\hline R (Renal insufficiency) & Niereninsuffizienz & $\begin{array}{l}\text { Kreatinin-Clearance <40 ml/min oder Kreatininkonzentration } \\
\text { im Serum }>177 \mu \mathrm{mol} / 1\end{array}$ \\
\hline A (Anemia) & Anämie & $\begin{array}{l}\text { Hämoglobin }<100 \mathrm{~g} / \mathrm{l} \text { oder }>20 \mathrm{~g} / \mathrm{l} \text { unter der unteren Grenze des } \\
\text { Normwertes }\end{array}$ \\
\hline B (Bone lesions) & Knochenläsionen & $\begin{array}{l}\text { Eine oder mehrere lytische Knochenläsionen im Röntgenbild, } \\
\text { in der CT oder in der PET-CT }\end{array}$ \\
\hline
\end{tabular}

Entscheidend für die Prognose des multiplen Myeloms ist das Tumorstadium bei der Diagnosestellung. Tabelle 3 zeigt die Stadieneinteilung des multiplen Myeloms gemäss dem revidierten Internal Staging System (ISS) von 2015 [6]. Die Einteilung in drei Stadien erfolgt aufgrund der Serumkonzentration an Albumin und $\beta 2$-Mikroglobulin, Laktatdehydrogenase $(\mathrm{LDH})$ und zytogenetischer Untersuchungen. Als prognostisch ungünstig gelten eine $\beta 2$ Mikroglobulin-Konzentration im Serum $>5.5 \mathrm{mg} / \mathrm{l}$ und ein erhöhte LDH-Aktivität. Mittels Fluoreszenz-in-Situ-Hybridisierung kann man beim überwiegenden Teil der Patienten mit multiplem Myelom chromosomale Aberrationen feststellen. Die Translokationen $\mathrm{t}(4 ; 14)$ und $\mathrm{t}(14 ; 16)$ sowie die Deletion $17 \mathrm{p}$ werden mit einer deutlich niedrigeren 5-JahresÜberlebensrate assoziiert [6]. 
Tab 3. Revised International Staging System (ISS) für multiples Myelom [6].

\begin{tabular}{|c|c|c|}
\hline Stadium & Definition & 5-Jahres-Überlebensrate (\%) \\
\hline I & $\begin{array}{l}\text { Albumin im Serum } \geq 3.5 \mathrm{~g} / \mathrm{dl} \\
\beta 2 \text {-Mikroglobulin im Serum }<3.5 \mathrm{mg} / \mathrm{l} \\
\text { Keine Hochrisiko-Zytogenetik und LDH normal }\end{array}$ & 82 \\
\hline II & Weder I noch III & 62 \\
\hline III & $\begin{array}{l}\beta 2-\text { Mikroglobulin im Serum }>5.5 \mathrm{mg} / \mathrm{l} \\
\text { Hochrisiko-Zytogenetik }[\mathrm{t}(4 ; 14), \mathrm{t}(14 ; 16) \text { oder del }(17 \mathrm{p})] \\
\text { oder LDH erhöht }\end{array}$ & 40 \\
\hline
\end{tabular}

\section{Frage 3}

Welche der folgenden Aussagen zu Laboruntersuchungen im Rahmen einer monoklonalen Gammopathie ist richtig? (Einfachauswahl, 1 richtige Antwort)

a) Eine unauffällige Serumprotein-Elektrophorese schliesst das Vorliegen einer monoklonalen Gammopathie aus.

b) Eine unauffällige Immunfixation des Serums schliesst das Vorliegen einer monoklonalen Gammopathie aus.

c) Der Nachweis einer homogenen Bande mit Anti-Leichtketten-Antikörpern in der Immunfixation beweist eine monoklonale Leichtketten-Gammopathie.

d) Die quantitative Bestimmung der Immunglobuline eignet sich nicht für die Quantifizierung eines M-Proteins.

e) Ein positives Proteinfeld auf dem Urinteststreifen bei einem Patienten mit multiplem Myelom weist auf die Ausscheidung von Bence-Jones-Protein hin.

\section{Antwort d) ist richtig}

\section{Kommentar}

Bei der Serumprotein-Elektropherese werden die Serumproteine gruppenweise auf einem Agarosegel entsprechend ihrer Ladung aufgetrennt und anschliessend densitometrisch ausgewertet. Eine monoklonale Gammopathie zeigt sich üblicherweise als ein scharf definiertes Band in der Gamma-Fraktion des Serumprotein-Elektrophoresegels. Die Sensitivität der Serumprotein-Elektropherese zur Erkennung einer monoklonalen Gammopathie wird mit etwa $80 \%$ angegeben [4]. $\mathrm{Zu}$ berücksichtigen ist, dass tiefkonzentrierte monoklonale Immunglobuline (wie z.B. monoklonale Leichtketten und monoklonales IgD oder IgE) abhängig von der Beweglichkeit im elektrischen Feld verdeckt werden können. Ausserdem kommt es vor, dass monoklonale Immunglobuline, insbesondere monoklonale IgA, nicht in der Gamma-Fraktion, sondern in der Beta- oder Alpha-Fraktion 
migrieren und von anderen Serumproteinen maskiert werden. Auch beim seltenen nichtsekretorischen multiplen Myelom und Amyloidose ist die Serumprotein-Elektrophorese in der Regel unauffällig. Deswegen sollten bei einer unauffälligen Serumprotein-Elektrophorese und Verdacht auf eine monoklonale Gammopathie zusätzlich eine Immunfixation und die Bestimmung der freien Leichtketten im Serum erfolgen.

Die Immunfixation im Serum ermöglicht durch den Einsatz von Antiseren gegen Immunglobulin-Komponenten die Klassifizierung und Typisierung monoklonaler Gammopathien. Gegenüber der Serumprotein-Elektrophorese weist die Immunfixation eine höhere Sensitivität auf [4]. Wenn in der Immunfixation keine homogenen Banden sichtbar sind, ist eine monoklonale Plasmazell-proliferative Erkrankung bei entsprechender Klinik nicht ausgeschlossen. Patienten mit nicht-sekretorischem multiplem Myelom weisen keine durch Immunfixation nachweisbare monoklonale Gammopathie auf. Zudem verhalten sich monoklonale Immunglobuline gelegentlich wie Kryoglobuline, das heisst sie präzipitieren in der Kälte. Eine nicht erkannte Kryoglobulinämie kann zu falsch-negativen-Befunde führen. Aggregierte Immunglobuline bleiben an der Auftragsstelle der Immunfixation liegen. Der Zusatz von $\beta$-Mercaptoethanol zur Patientenprobe führt zur Auflösung der Aggregate, sodass die Immunfixation bewertbar wird. Schliesslich bleibt bei einem starken Antigenüberschuss in der Patientenprobe die Immunpräzipitation im zentralen Bandenbereich aus. Dieses sogenannte Prozonenphänomen kann durch Wiederholung der Immunfixation mit einem verdünnten Ansatz eliminiert werden.

Für die Immunfixation im Serum werden normalerweise spezifische Antikörper, welche gegen Schwerketten vom Typ IgG, IgA und IgM sowie gegen Leichtketten vom Typ Kappa und Lambda gerichtet sind, eingesetzt. Monoklonale Gammopathien vom IgD- oder IgESubtyp werden mittels der routinemässig durchgeführten Immunfixation nicht erfasst. Falls in der Immunfixation nur eine monoklonale Leichtkette nachweisbar ist, sollte demzufolge das Vorliegen eines IgE- oder IgD-M-Proteins geprüft werden.

Eine quantitative Bestimmung der Immunglobuline im Serum mittels Immunnephelometrie oder Immunturbidimetrie ist für die Quantifizierung eines M-Proteins nicht brauchbar. Der direkte Vergleich zwischen Serumprotein-Elektrophorese und Immunnephelometrie oder Immunturbidimetrie zeigt erhebliche Diskrepanzen bei der quantitativen Bestimmung von monoklonalen Immunglobulinen im Serum; mit der Immunnephelometrie oder Immunturbidimetrie finden sich im allgemeinen höhere Werte [4]. Die genaue Ursache ist nicht bekannt. Dementsprechend sollte die absolute Konzentration des M-Proteins anhand der Serumprotein-Elektrophorese berechnet werden. Nephelometrische oder turbidimetrische 
Verfahren zur Bestimmung der Immunglobulin-Klassen können bei Verdacht eines myelombedingten sekundären Antikörpermangels eingesetzt werden. Vor kurzem wurde einen neuen Test (Hevylite®) entwickelt, der die separate Messung der verschiedenen Leichtkettentypen jeder einzelnen Immunglobulinklasse ( $\operatorname{IgG} \kappa, \operatorname{IgG} \lambda$, $\operatorname{Ig} A \kappa, \operatorname{Ig} A \lambda, \operatorname{Ig} M \kappa$ und $\operatorname{Ig} M \lambda$ ) ermöglicht [7]. Dieser neue Test ist im Vergleich zur Serumprotein-Elektrophorese und Immunfixation weniger aufwändig und erlaubt zudem gleichzeitig die Charakterisierung und Quantifizierung von M-Komponenten. In der Schweiz gibt es zurzeit noch keine offiziellen Empfehlungen für den Einsatz dieses Messverfahrens.

Mit dem Urin ausgeschiedene freie Leichtketten werden als Bence-Jones-Proteine bezeichnet. Der in der Routinediagnostik eingesetzte Urinteststreifen eignet sich nicht für den Nachweis von Bence-Jones-Proteinen im Urin. Bence-Jones-Proteine werden mit den üblichen Urinteststreifen nicht erfasst. Zur Diagnostik einer Bence-Jones-Proteinurie ist eine Immunfixation im 24 h-Sammelurin oder morgendlichen Spontanurin erforderlich.

\section{Frage 4}

Welche Aussagen über die Bestimmung von freien Leichtketten im Serum sind richtig? (Mehrfachauswahl, mehrere Antworten richtig)

a) Die Bestimmung der freien Leichtketten im Serum wird bei der initialen Abklärung einer monoklonalen Gammopathie empfohlen.

b) Die Konzentration freier Leichtketten vom Typ Kappa ( $\kappa)$ ist im Serum niedriger als die Konzentration freier Leichtketten vom Typ Lambda $(\lambda)$.

c) Eine erhöhte Konzentration der freien Leichtketten deutet zwingend auf die Produktion eines monoklonalen Proteins hin.

d) Der $\kappa / \lambda$-Quotient hängt nicht von der Nierenfunktion ab.

e) Aufgrund der langen Halbwertszeit der freien Leichtketten im Serum, lässt sich ein Therapieerfolg erst nach einer längeren Zeit abschätzen.

\section{Antworten a) und b) sind richtig}

\section{Kommentar}

Der Test zur Bestimmung der freien Leichtketten (Free light chains, FLC) im Serum ist ein nephelometrischer Immunoassay, welcher selektiv die nicht an Schwerketten gebundenen (freien) Kappa $(\kappa)$ - und Lambda $(\lambda)$-Leichtketten quantifiziert. Dazu werden Antikörper mit einer Spezifität gegen Epitope der Leichtketten, die im intakten Immunglobulinmolekül verborgen sind, angewendet [5]. Proliferative Tumore der Lymphozyten der B-Linie sind 
üblicherweise mit einer übermässigen Produktion an FLC assoziiert. In $95 \%$ aller symptomatischen Myelomfälle findet man eine Erhöhung der FLC-Konzentration im Serum [5]. Hohe FLC-Werte alleine beweisen aber nicht, dass eine monoklonale Produktion vorliegt. Erhöhte Serumkonzentrationen an FLC können auch im Rahmen von chronischen Infektionen, Autoimmunerkrankungen, wie Lupus erythematodes und rheumatoide Arthritis, und insulinabhängigem Diabetes auftreten [8]. Dagegen ist ein abnormales Verhältnis von $\kappa$ $\mathrm{zu} \lambda$-Leichtketten (kleiner als 0.26 oder grösser als 1.65) ein starker Indikator für eine monoklonale Immunglobulinvermehrung [4]. Bei polyklonalen Gammopathien ist der $\kappa / \lambda$ Quotient im Normalbereich.

FLC werden auch unter physiologischen Bedingungen im leichten Überschuss von den BZellen gebildet; pro Tag 0.5 bis $1 \mathrm{~g}$ und etwa doppelt so viele $\kappa$-Leichtketten wie $\lambda$ Leichtketten [4]. Bei normaler Nierenfunktion werden FLC glomerulär filtriert und anschliessend von den proximalen Tubuluszellen rückresorbiert und abgebaut. Da $\kappa$-FLC hauptsächlich als Monomere vorliegen und sie somit im Vergleich zu $\lambda$-FLC, die meistens zu einem Dimer verbunden sind, einfacher glomerulär filtriert werden, ist die Konzentration der $\kappa$-FLC im Serum normalerweise niedriger als die Konzentration der $\lambda$-FLC [4]. Bei niereninsuffizienten Patienten erfolgt der Abbau von FLC wegen der eingeschränkten glomerulären Filtration vermehrt durch die Zellen des retikuloendothelialen Systems. Die Elimination über das retikuloendotheliale System wird jedoch nicht vom Molekulargewicht der FLC beeinflusst und es kommt daher zu einer Zunahme der $\kappa / \lambda$-Ratio [9]. Entsprechend gelten für Patienten mit Niereninsuffizienz modifizierte Normalwerte.

Gemäss den aktualisierten Empfehlungen zur Diagnostik und Behandlung von multiplem Myelom in der Schweiz sollte eine FLC-Bestimmung im Serum und die Ermittlung des $\kappa / \lambda$ Ratio bei jedem Patienten Bestandteil der initialen Abklärung einer monoklonalen Gammopathie sein [10]. Stark veränderte $\kappa / \lambda$-Quotienten $(<0.03$ oder $>32)$ korrelieren mit einer schlechteren Prognose bezüglich dem Gesamtüberleben nach 1 Jahr und 5 Jahren [5]. Des Weiteren erlaubt die Bestimmung der FLC im Serum, aufgrund deren kurzen Halbwertszeit von 2 bis 6 Stunden, die Wahrscheinlichkeit eines therapeutischen Erfolges rasch einzuschätzen und ein Rezidiv frühzeitig zu erkennen [5]. 


\title{
Korrespondenzadresse
}

\author{
Dr. Wijtske Annema
}

Institut für Klinische Chemie

Universitätsspital Zürich

Rämistrasse 100

8091 Zürich

wijtske.annema@usz.ch

\section{Bibliografie}

1. Kyle RA, Rajkumar SV: Monoclonal gammopathy of undetermined significance. Br J Haematol 2006; 134 : 573-589.

2. Cairoli A, Duchosal MA: Multiples Myelom: Diagnostik und therapeutische Perspektive. Schweiz Med Forum 2013; 13: 746-751.

3. Rajkumar SV, Dimopoulos MA, Palumbo A, et al.: International Myeloma Working Group updated criteria for the diagnosis of multiple myeloma. Lancet Oncol 2014; 15: e538-e548.

4. Thomas L: Labor und Diagnose: Monoklonale Plasmazell-proliferative Erkrankungen Bd.2, 8. Aufl. Frankfurt/Main. Th-Books Verl.-Ges.; 2012: 1374-1396.

5. Rivier D: Freie Immunglobulin-Leichtketten und Gammopathien. Schweiz Med Forum 2012; 12: 585-592.

6. Palumbo A, Avet-Loiseau H, Oliva S, et al: Revised International Staging System for multiple myeloma: a report from International Myeloma Working Group. J Clin Oncol 2015; 33: 2863-2869.

7. Bernasconi L, Mundwiler E: Monoklonale Gammopathien. Neue IMWG-Kriterien und Labortests für die Diagnose, Prognose und Verlaufskontrolle des Multiplen Myeloms. Pipette - Swiss Laboratory Medicine 2016; 1: 11-13.

8. Aubert V, Duchosal MA: Monoklonale Gammopathien: Anwendung des Freie-Leichtketten-Serumtest. Schweiz Med Forum 2012; 12: 576-577.

9. Hutchison CA, Harding S, Hewins P, et al: Quantitative assessment of serum and urinary polyclonal free light chains in patients with chronic kidney disease. Clin J Am Soc Nephrol 2008; 3: 1684-1690.

10. Samaras P, Bargetzi M, Betticher DC, et al: Current status and updated recommendations for diagnosis and treatment of plasma cell myeloma in Switzerland. Swiss Med Wkly 2015; 145 :w14100. 